\title{
Poly(ADP-Ribose) Polymerase-1: A Novel Therapeutic Target in Necrotizing Enterocolitis
}

\author{
PETER J. GIANNONE, ALICIA A. ALCAMO, BRANDON L. SCHANBACHER, CRAIG A. NANKERVIS, GAIL E. BESNER, \\ AND JOHN A. BAUER
}

Center for Perinatal Research [P.J.G., A.A.A., B.L.S., C.A.N., G.E.B., J.A.B.], The Research Institute at Nationwide Children's Hospital, Columbus, Ohio 43205; Department of Pediatrics [P.J.G., C.A.N., J.A.B.], Department of Surgery [G.E.B.], The Ohio State University, Columbus, Ohio 43205

\begin{abstract}
Necrotizing enterocolitis (NEC) is the most common gastrointestinal disease of infancy, afflicting $11 \%$ of infants born 22-28 wk GA. Both inflammation and oxidation may be involved in NEC pathogenesis through reactive nitrogen species production, protein oxidation, and DNA damage. Poly(ADP-ribose) polymerase-1 (PARP-1) is a critical enzyme activated to facilitate DNA repair using nicotinamide adenine dinucleotide $(\mathrm{NAD}+)$ as a substrate. However, in the presence of severe oxidative stress and DNA damage, PARP-1 overactivation may ensue, depleting cells of NAD+ and ATP, killing them by metabolic catastrophe. Here, we tested the hypothesis that NO dysregulation in intestinal epithelial cells during NEC leads to marked PARP-1 expression and that administration of a PARP-1 inhibitor (nicotinamide) attenuates intestinal injury in a newborn rat model of NEC. In this model, $56 \%$ of control pups developed NEC (any stage) versus 14\% of pups receiving nicotinamide. Forty-four percent of control pups developed high-grade NEC (grades 3-4), whereas only $7 \%$ of pups receiving nicotinamide developed high-grade NEC. Nicotinamide treatment protects pups against intestinal injury incurred in the newborn rat NEC model. We speculate that PARP-1 overactivation in NEC may drive mucosal cell death in this disease and that PARP-1 may be a novel therapeutic target in NEC. (Pediatr Res 70: 67-71, 2011)
\end{abstract}

$\mathrm{N}^{\mathrm{c}}$ ecrotizing enterocolitis (NEC) is the most common gastrointestinal disease of infancy, with population studies estimating the incidence of NEC at between 0.3 and 2.4 per 1000 live births in the United States and afflicting up to $11 \%$ of all infants born 22-28 wk gestation $(1,2)$. Mortality rates range from $20 \%$ to $44 \%$ in infants with birth weights $<1500$ $\mathrm{g}$ and up to $20 \%$ in infants $>2500 \mathrm{~g}$ (3). Although first described in the 1800s, the mechanistic basis of NEC remains poorly understood despite years of basic science and clinical exploration (4). Factors in the newborn intestine modulating the innate immune response as well as the protective and toxic effects of the free radical NO have been postulated as possible contributors to the initiation and/or progression of NEC $(5,6)$.

Endogenous NO is generated during the enzymatic conversion of L-arginine to L-citrulline and is catalyzed by a family of enzymes known as NO synthases (NOS) $(7,8)$. Three NOS isoenzymes function in the gastrointestinal tract to produce

Received October 21, 2010; accepted January 4, 2011.

Correspondence: Peter J. Giannone, M.D., Center for Perinatal Research, The Research Institute at Nationwide Children's Hospital, 700 Children's Drive, Columbus, OH 43205; e-mail: peter.giannone@ nationwidechildrens.org

Supported, in part, by NIH Grant 1K23DK078909.
NO: neuronal NOS (NOS1), inducible NOS (NOS2), and endothelial NOS (NOS3). The synthase most commonly implicated in NO dysregulation is NOS2 (9). Excess or uncontrolled production of $\mathrm{NO}$ can shift it from a useful cellular signal to a toxic free radical (10). NO dysregulation promotes the formation of peroxynitrite, a highly reactive nitrogen species known to nitrate protein tyrosine residues and cause cellular oxidative damage to organelles and DNA (11).

Widespread NOS2 induction and protein nitration have been found in human NEC specimens (12). After DNA damage due to oxidation, poly(ADP-ribose) polymerase-1 (PARP-1) is a critical enzyme activated to facilitate DNA repair (Fig. 1). This enzyme uses NAD + (nicotinamide adenine dinucleotide) as a substrate and attaches multiple ADPribose (PAR) units to itself and other acceptor proteins $(13,14)$. This poly(ADP-ribosyl)ation allows the acceptor proteins to selectively influence important cellular responses that enhance DNA repair (15). However, in the presence of severe cellular oxidative stress and DNA damage, overactivation of PARP-1 may ensue (16). This may lead to cell death by two possible mechanisms. First, significant PARP-1 activation may deplete the cells of NAD+/ATP, killing the cells by metabolic catastrophe (16). Cells with already low NAD+ stores may be more prone such as proliferating enterocytes in the crypts or cells entering apoptosis such as those at the villus tips (17). Second, in the presence of adequate cell energy stores, increased PARP-1 activation can lead to apoptosis via apoptotic inducing factor (AIF) from the mitochondria or by caspase-dependant mechanisms (18).

The importance of apoptosis during the evolution of NEC has been supported in multiple experiments (19-23). However, at some point, necrosis will ensue whether the disease continues to progress. Thus, a mediator such as PARP-1 in an injured or activated cell may serve as a checkpoint or governor between the fate of cellular repair and the fate of cell death (via either apoptosis or necrosis) and may play a vital role in disease progression including necrotizing enterocolitis. Our objective was to test the hypothesis that $\mathrm{NO}$ dysregulation in the intestinal epithelial cell during NEC leads to marked

Abbreviations: 3-NT, 3-nitrotyrosine; AG, aminoguanidine; IOD, integrated optical density; LPS, lipopolysaccharide; NEC, necrotizing enterocolitis; NOS2, inducible NO synthase; PARP-1, poly(ADP-ribose) polymerase-1 


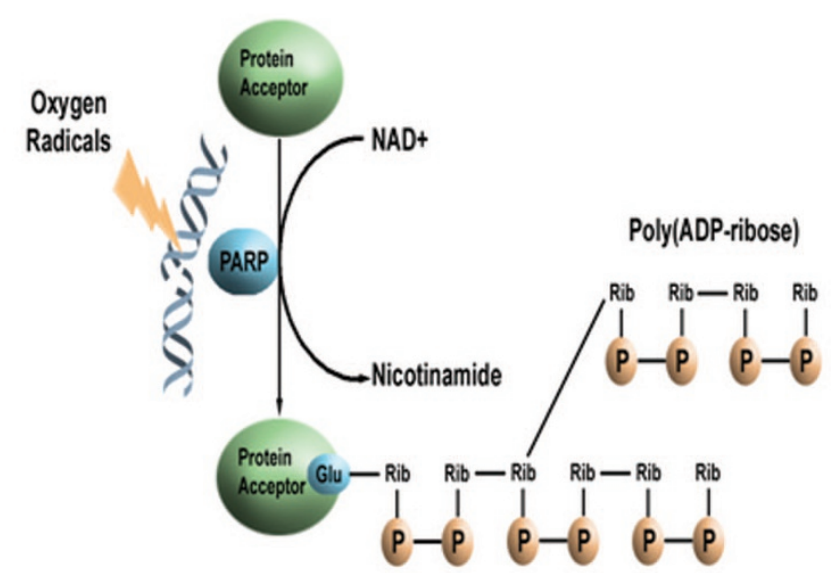

Figure 1. Illustration of PARP-1 action after DNA damage. PARP-1 adds PAR units onto DNA repair proteins to facilitate DNA repair. This action requires NAD + and ATP. Overactivation of PARP-1 may deplete the cell of NAD + and ATP stores. Adapted from Schreiber et al. Nat Rev Mol Cell Biol 7:517, copyright () 2006 MacMillan Publishers Ltd., with permission.

PARP-1 expression, and that administration of a known PARP-1 inhibitor (nicotinamide) will attenuate intestinal injury in a newborn rat model of NEC.

\section{METHODS}

Animals. Pregnant Sprague-Dawley rats (Harlan Laboratories Indianapolis, IN) were obtained at gestation E16 and were allowed access to standard chow and water ad libitum until delivery of the pups via C-section at E21 (term). All animal studies were approved by The Research Institute at Nationwide Children's Hospital's Institutional Animal Care and Use Committee (IACUC).

Neonatal rat NEC model. After delivery, rat pups were placed on the rat NEC model protocol as previously described $(24,25)$. Each pup was given enteral LPS (1 mg/kg E. coli lipopolysaccharide) followed by enteral orogastric artificial feedings six times a day. The feedings consisted of $15 \mathrm{~g}$ of Similac 60/40 powder (Ross Pediatrics, Columbus, OH) added to $75 \mathrm{~mL}$ Esbilac Liquid Milk Replacer (Pet-Ag, New Hampshire, IL) to provide 200 $\mathrm{kcal} / \mathrm{kg} / \mathrm{d}$. Starting within 2-3 h of delivery, pups were exposed to hypoxia (100\% nitrogen) for $2 \mathrm{~min}$ followed immediately by exposure to hypothermia $\left(10 \mathrm{~min}\right.$ at $\left.4^{\circ} \mathrm{C}\right)$ twice a day. Pups were killed at $4 \mathrm{~d}$ of age or sooner if they developed evidence of distress, including increased work of breathing or lethargy. After sacrifice, the intestines were removed and placed in $10 \%$ formalin for $24 \mathrm{~h}$ followed by saline. After fixation of the intestines with formalin, the intestine was placed in paraffin block, and histologic sections were prepared and stained with hematoxylin and eosin for further analysis.

Grading of intestinal injury. A previously established grading system was used to objectively determine the degree of observed intestinal injury $(25,26)$. The grading system was as follows: grade 0 , no evidence of intestinal injury; grade 1, sloughing at the tips of the villi only; grade 2, sloughing to the mid villous; grade 3, necrosis of the entire villus; and grade 4, necrosis of the intestinal wall that includes the entire villus extending into the submucosa. Two separate investigators blinded to the study graded each intestinal sample.

Enteral administration of NOS2 blocker or nicotinamide. The selective NOS2 inhibitor aminoguanidine (AG; $10 \mathrm{mg} / \mathrm{kg} / \mathrm{d}$; Cayman Chemical, Ann Arbor, MI) $(n=11)$ or vehicle (sterile water) $(n=12)$ was administered per orogastric tube before the first feeding and then daily throughout the 4-day protocol in animals in the aminoguanidine group. In a separate experiment, the PARP inhibitor nicotinamide $(500 \mathrm{mg} / \mathrm{kg} / \mathrm{d}$; Sigma Chemical Co.-Aldrich, St. Louis, MO) $(n=14)$ or vehicle (sterile water) $(n=16)$ was administered per orogastric tube before the first feeding and then daily throughout the 4-day protocol in animals in the nicotinamide group. AG and nicotinamide were suspended in sterile water for these experiments.

Human NEC sample collection. Human intestinal tissues obtained during operative procedures carried out at Nationwide Children's Hospital (Columbus, $\mathrm{OH}$ ) were used. Sample collection was approved by Nationwide Children's Hospital Institutional Review Board. The operative procedures that provide tissue for this study occurred during the course of standard care for intestinal disease states (i.e. intestinal obstruction or NEC). The pathologist first examined the tissue and those portions required to make a pathologic diagnosis are placed in the proper fixative; the remaining tissue that normally would be discarded by the pathologist was then analyzed in this study. The sample is cut into two pieces. One piece is placed in $10 \%$ formalin for $24 \mathrm{~h}$ followed by saline, and the second was immediately frozen at $-80^{\circ} \mathrm{C}$ for future use. For the purposes of this study, we used NEC specimens taken from the region of the resection margin. These tissues were not grossly necrotic and had evidence of viable proximal mucosa.

Intestinal immunohistochemistry. Five-micrometer sections from formalin-fixed paraffin-embedded ileal tissues from either human or rat species were mounted on slides, rehydrated in a graded series of alcohols, and deparaffinized for immunohistochemical staining. Immunohistochemistry was performed using primary antibodies specific for NOS2 (Transduction Labs; 1:200), 3-nitrotyrosine (3-NT) (Millipore, Billerica, MA; 1:400), and PARP-1 (Trevigen, Helgerman, CT 1:1000), followed by biotinylated universal goat link secondary antibody (Biocare Medical, Concord, CA), followed by streptavidin-HRP (Biocare Medical) and diaminobenzadine (DAB; Sigma Chemical Co.-Aldrich) as the chromogen. Tissue sections were visualized with an Olympus Optical (Melville, NY) BX-40 microscope (40× objective). Images were captured under identical lighting conditions and optical settings and analyzed using image analysis software (Image Pro Plus; Media Cybernetics, Silver Springs, MD). In each image, two transmural regions (distal villus and proximal villus) were outlined and the staining intensities, measured as integrated OD (IOD), were determined for each region.

Image analysis. Images were captured using a research grade Olympus BX-40 microscope, a high-resolution color digital camera (Diagnostic Instruments, Sterling Hts, MI) and a Pentium 233 PC workstation, and analyzed using Image Pro Plus software (Media Cybernetics, MD). Separate methods have been developed that allow semiquantitative determination of the extent of immunoreactivity of sections. For tissues exhibiting a more punctate staining pattern (commonly observed with NOS2 presence), the image analysis software provided an automated analysis tool that allows for counting of dark or light objects; these objects can be gated to discriminate very small or large (i.e. noncellular) debris based on pixel size. Images are segmented after extraction of the blue channel and conversion to gray scale as previously described $(27,28)$. We have previously used this method to quantify NOS2 positive cells in a cross section of ileum from newborn rats in a model of prenatal LPS exposure and also hyperoxia exposure $(25,27,28)$.

The degree of 3-NT, NOS2, and PARP-1 staining in the mucosa of ileal tissues from rats with grades 1 and 2 injury were analyzed. Focus was placed on these grades to represent the early transition to severe tissue injury and cell death. Moreover, the mucosal region is obliterated in severe NEC (Fig. 2) (grades 3 and 4 injury in the newborn rat model). Thus, the proximal mucosa could not be evaluated in these grades of injury.

Statistical analyses. Data are expressed as means \pm SEM. Comparative analyses were completed by $t$ test and two-way ANOVA with Bonferroni posttests to determine relative differences among our study groups. A $p$ value $<0.05$ was considered significant.

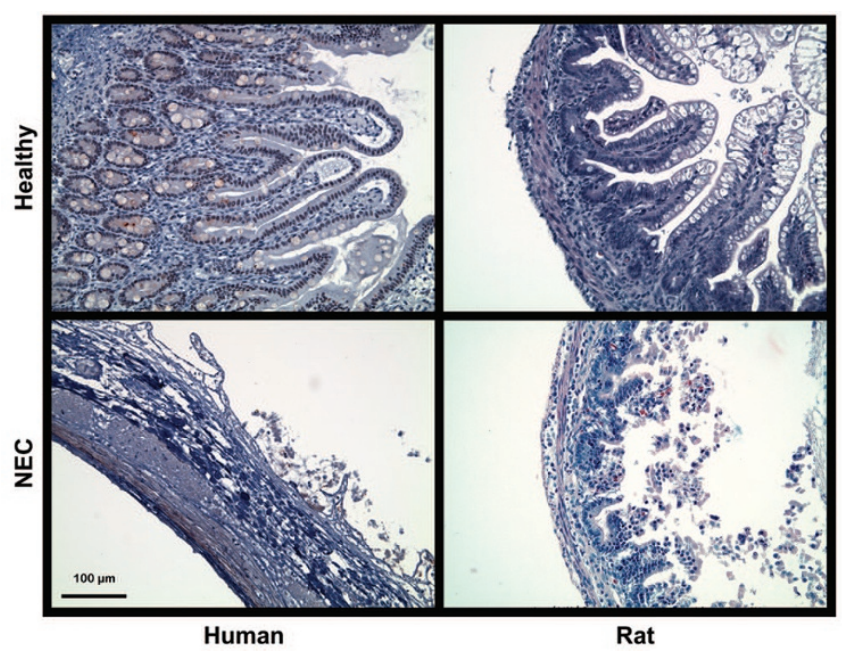

Figure 2. Comparison of human and rodent NEC. Shown are representative photomicrographs of both human intestinal tissues and rat intestinal tissues with NEC. The upper panels show healthy tissue in both species and the lower panels shows NEC in both species. These data support the validity of the use of rodents as in vivo models of NEC. Scale bar $=100 \mu \mathrm{m}$, magnification $\times 40$. 

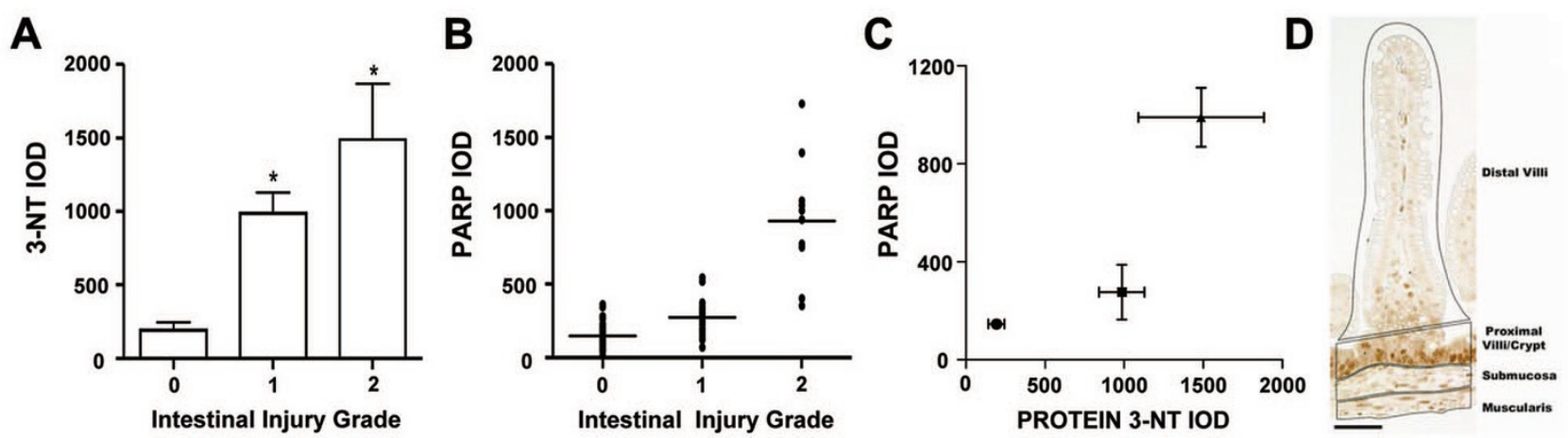

Figure 3. 3-NT and PARP-1 expression in experimental NEC. (A) 3-Nitrotyrosine staining in the proximal mucosa of pups exposed to experimental NEC. 3-NT staining increased as the degree of injury increased in this region ( $*=p<0.05 v s$ control; two-way ANOVA). (B) PARP immunostaining is significantly increased in the proximal mucosa as severity of injury increases. (C) PARP activation correlates with areas of greatest nitrative injury in the proximal mucosa. $\boldsymbol{\bullet}$, Control; $\boldsymbol{\square}$, grade 1 NEC; $\boldsymbol{\Delta}$, grade 2 NEC. $(D)$ Representation of areas of mucosa discussed. Scale bar $=50 \mu$, magnification $\times 40$.
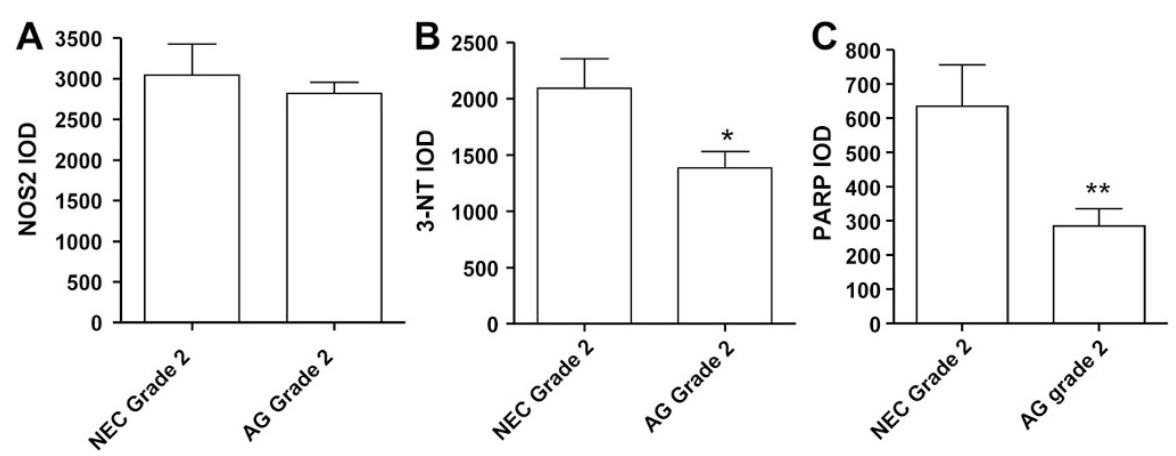

Figure 4. Effects of aminoguanidine on NOS2, 3-NT, and PARP-1 expression in experimental NEC. (A) NOS2 immunostaining was unchanged. In pups with grade 2 NEC, there was significantly less 3-NT $(B)$ and PARP $(C)$ immunostaining within the proximal mucosa of pups treated with the NOS2 inhibitor aminoguanidine. $*=p<0.05, * *=p<0.005$ vs NEC grade 2 .
Table 1. Effect of nicotinamide on experimental NEC

\begin{tabular}{lccc}
\hline & Control & Nicotinamide & $p$ \\
\hline Percentage of NEC & $56 \%$ & $14 \%$ & $\leq 0.01$ \\
Percentage of NEC $\geq$ grade 2 & $44 \%$ & $7 \%$ & $<0.02$ \\
\hline
\end{tabular}

Pups treated with nicotinamide have significantly less evidence of any intestinal injury than controls when exposed to the rat model of NEC ( $p<$ $0.05 ; t$ test). Pups treated with nicotinamide tended to have less severe intestinal injury $(p<0.05 ; t$ test $)$.

\section{RESULTS}

Evidence of reactive nitrogen species formation in the rat NEC model. Digital image analysis showed evidence of increased 3-NT in the proximal mucosal region (Fig. 3A) in tissues with grade 1 or 2 injury, demonstrating an increased prevalence of nitrative cellular injury.

PARP-1 activation in the rat NEC model. We observed significant increases in PARP-1 in the same regions of tissue in which we observed protein nitration (Fig. $3 B$ ). In addition, there was a positive association of PARP-1 prevalence in the regions of highest protein nitration among the NEC tissues investigated (Fig. 3C).

Inhibition of NOS2 reduces intestinal mucosal PARP-1 expression. NOS2 expression was unchanged with aminoguanidine treatment in the proximal mucosa. In contrast, 3-NT and PARP expression were significantly decreased in the proximal mucosa of pups treated with aminoguanidine (Fig. 4). Although there was no significant difference in overall NEC between treatment and control, severe grades of injury were protected against ( $0 \%$ versus $50 \%$; data not shown). These finding are consistent with those of other investigators (12).

Nicotinamide is protective against intestinal injury in a newborn rat model of NEC. Administration of nicotinamide was protective against the development of any NEC $(p<$ $0.01)$ and also protected against more severe grades of injury $(p<0.02$; Table 1).

PARP-1 expression is elevated in human intestinal tissue resected for NEC. Figure $5 A$ shows PARP-1 immunostaining in a specimen of small intestine from an infant with bowel obstruction, whereas Figure $5 B$ shows PARP-1 immunostaining in intestinal tissue resected from an infant with NEC. Note this is not severe NEC or markedly necrotic bowel because the mucosa is still present in this portion of the specimen. When quantified $(n=17$ for control with mean GA $29 \pm 4 \mathrm{wk}$ and $n=20$ for diseased with mean GA $31 \pm 5$ wk), PARP-1 immunostaining was significantly increased in the intestinal mucosa of human NEC specimens (Fig. 5B).

\section{DISCUSSION}

Our objective was to test the hypothesis that NO dysregulation in the intestinal epithelial cell during NEC leads to marked PARP-1 expression, and that treatment with a known PARP-1 inhibitor attenuates intestinal injury in a newborn rat model of NEC. Analyses revealed evidence of increased 3-NT in the proximal mucosal region, demonstrating an increased prevalence of nitrative cellular injury. PARP-1 immunostaining was significantly decreased in pups treated with AG and 


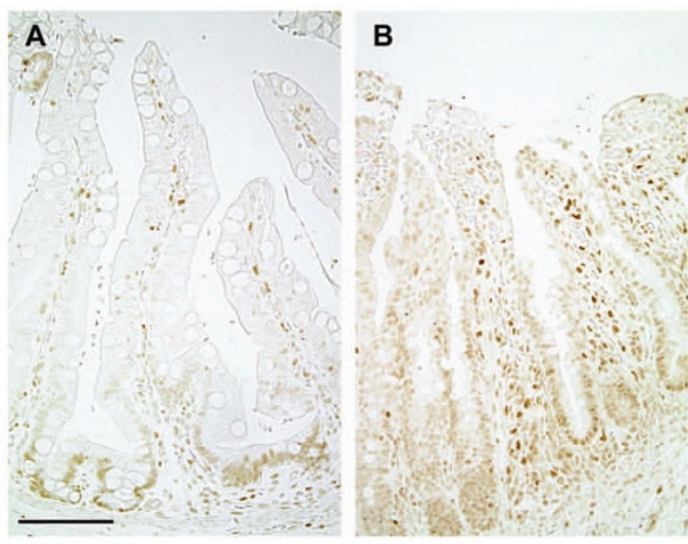

Figure 5. PARP-1 expression in human NEC. Shown are representative photomicrographs of PARP-1 immunostaining in healthy newborn small intestine $(A)$ and an intestinal specimen resected for NEC $(B)$. PARP is represented by the brown staining, particularly prominent in the mucosa of the NEC specimen. Scale bar $=100$ $\mu \mathrm{m}$, magnification $\times 40$. (C) Quantitation of PARP-1 immunostaining in NEC $v s$ control tissues. $*=p<0.01$ vs control. subjected to experimental NEC. It seems that PARP-1 activation is downstream from NOS2 induction, and that blocking the NOS2 enzyme can reduce mucosal PARP in this setting. These data strongly support the concepts developed in other disease states, specifically that reactive nitrogen species lead to PARP-1 activation. Note that the use of amioguanidine will not decrease transcription of NOS2 protein, but it will decrease the enzyme activity. Thus, the downstream effects of the enzyme are attenuated as we have demonstrated in these experiments and have been shown previously by Vicente et al. (29) as well as others. PARP-1 is a contributor to both disease progression and severity in other disease states including myocardial infarction and inflammatory bowel disease (30-32). PARP-1 is therapeutic target in these disease states as well as others (33). Therefore, it is possible that treatment with a PARP-1 inhibitor may also attenuate intestinal injury in NEC.

On administration of the known PARP-1 inhibitor nicotinamide in our rat NEC model, both intestinal injury and PARP-1 expression were significantly decreased. Nicotinamide was chosen in these initial experiments because of its wide safety profile and known PARP-1 inhibitory effects (16). There are several other PARP-1 inhibitors in clinical trials for other disease states, including diabetes and cancer (33). However, nicotinamide does have other effects, such as attenuation of inflammation, which may also make it an ideal choice for a therapeutic in premature infants. These experiments demonstrate evidence that PARP-1 may play a role in newborn intestinal injury, and inhibition of PARP-1 may be protective against NEC. However, because of other potential roles, nicotinamide might play in protecting the newborn intestine from injury; our finding supports our hypothesis but do not definitively prove PARP's mechanistic role in NEC. Other studies have identified the elevation of PARP-1 in experimental models of NEC, but they only identified it as a marker for cell death (34). Studies to putatively define the role of PARP-1 in NEC using PARP-1 "knock out" and "knock down" models of newborn intestinal injury are warranted and on-going in our laboratory as well as optimizing the dosing of nicotinamide and newborn intestinal injury.

Our results also support the premise that PARP-1 may play a role in the events leading to enterocyte cell death during human NEC. PARP-1 is significantly elevated in human NEC specimens when compared with control newborn intestinal tissues. Specifically, PARP-1 expression was elevated in the NEC surgical specimens that still have a relatively intact intestinal mucosa. It is generally accepted that the intestinal injury incurred in NEC begins in the mucosa and propagates from there $(25,35,36)$. This region of intestine is critical for restitution from the initial stages of NEC. If the diseased intestinal tissue was not removed, it is plausible that these tissues would have progressed to NEC because the majority of these samples were at the resection margins of a sample with a necrotic midsection. Therefore, it is reasonable to hypothesize that PARP-1 may be acting as a gate keeper for enterocyte cell death during NEC. Multiple studies have shown the importance of apoptosis and early cell death in the progression of bowel injury in NEC $(19,23)$. Identifying mediators that determine this early decision by the cells for restitution or cell death may allow us to develop therapies to halt the progression of this disease and subsequently decrease the significant morbidities associated with NEC (37-39).

In summary, PARP-1 may be an important effector of intestinal injury and cell death associated with NEC. Further exploration of its mechanistic role in NEC as well as its viability as a therapeutic target and its safety in future studies is warranted.

\section{REFERENCES}

1. Christensen RD, Gordon PV, Besner GE 2010 Can we cut the incidence of necrotizing enterocolitis in half-today? Fetal Pediatr Pathol 29:185-198

2. Stoll BJ, Hansen NI, Bell EF, Shankaran S, Laptook AR, Walsh MC, Hale EC, Newman NS, Schibler K, Carlo WA, Kennedy KA, Poindexter BB, Finer NN, Ehrenkranz RA, Duara S, Sánchez PJ, O'Shea TM, Goldberg RN, Van Meurs KP, Faix RG, Phelps DL, Frantz ID 3rd, Watterberg KL, Saha S, Das A, Higgins RD; Eunice Kennedy Shriver National Institute of Child Health and Human Development Neonatal Research Network 2010 Neonatal outcomes of extremely preterm infants from the NICHD Neonatal Research Network. Pediatrics 126:443-456

3. Fitzgibbons SC, Ching Y, Yu D, Carpenter J, Kenny M, Weldon C, Lillehei C, Valim C, Horbar JD, Jaksic T 2009 Mortality of necrotizing enterocolitis expressed by birth weight categories. J Pediatr Surg 44:1072-1075; discussion 1075-1076

4. Obladen M 2009 Necrotizing enterocolitis-150 years of fruitless search for the cause. Neonatology 96:203-210

5. Potoka DA, Nadler EP, Upperman JS, Ford HR 2002 Role of nitric oxide and peroxynitrite in gut barrier failure. World J Surg 26:806-811

6. Potoka DA, Nadler EP, Zhou X, Zhang XR, Upperman JS, Ford HR 2000 Inhibition of NF-kappaB by IkappaB prevents cytokine-induced NO production and promotes enterocyte apoptosis in vitro. Shock 14:366-373

7. Moncada S, Higgs A 1993 The L-arginine-nitric oxide pathway. N Engl J Med 329:2002-2012

8. Sessa WC 1994 The nitric oxide synthase family of proteins. J Vasc Res 31:131-143

9. Stuehr DJ, Marletta MA 1985 Mammalian nitrate biosynthesis: mouse macrophages produce nitrite and nitrate in response to Escherichia coli lipopolysaccharide. Proc Natl Acad Sci USA 82:7738-7742 
10. Pryor WA, Squadrito GL 1995 The chemistry of peroxynitrite: a product from the reaction of nitric oxide with superoxide. Am J Physiol 268:L699-L722

11. Beckman JS, Koppenol WH 1996 Nitric oxide, superoxide, and peroxynitrite: the good, the bad, and ugly. Am J Physiol 271:C1424-C1437

12. Zamora R, Bryan NS, Boyle P, Wong C, Milsom AB, Jaffe R, Feelisch M, Ford HR 2005 Nitrosative stress in an animal model of necrotizing enterocolitis. Free Radic Biol Med 39:1428-1437

13. Schreiber V, Dantzer F, Ame JC, de Murcia G 2006 Poly(ADP-ribose): novel functions for an old molecule. Nat Rev Mol Cell Biol 7:517-528

14. Szabo C, Pacher P, Swanson RA 2006 Novel modulators of poly(ADP-ribose) polymerase. Trends Pharmacol Sci 27:626-630

15. Meyer-Ficca ML, Meyer RG, Jacobson EL, Jacobson MK 2005 Poly(ADP-ribose) polymerases: managing genome stability. Int J Biochem Cell Biol 37:920-926

16. Szabó C 2006 Poly(ADP-ribose) polymerase activation by reactive nitrogen species-relevance for the pathogenesis of inflammation. Nitric Oxide 14:169-179

17. Hassa PO, Haenni SS, Elser M, Hottiger MO 2006 Nuclear ADP-ribosylation reactions in mammalian cells: where are we today and where are we going? Microbiol Mol Biol Rev 70:789-829

18. Kolthur-Seetharam U, Dantzer F, McBurney MW, de Murcia G, Sassone-Corsi P 2006 Control of AIF-mediated cell death by the functional interplay of SIRT1 and PARP-1 in response to DNA damage. Cell Cycle 5:873-877

19. Jilling T, Lu J, Jackson M, Caplan MS 2004 Intestinal epithelial apoptosis initiates gross bowel necrosis in an experimental rat model of neonatal necrotizing enterocolitis. Pediatr Res 55:622-629

20. Hunter CJ, Singamsetty VK, Chokshi NK, Boyle P, Camerini V, Grishin AV, Upperman JS, Ford HR, Prasadarao NV 2008 Enterobacter sakazakii enhances epithelial cell injury by inducing apoptosis in a rat model of necrotizing enterocolitis. J Infect Dis 198:586-593

21. Clark JA, Lane RH, Maclennan NK, Holubec H, Dvorakova K, Halpern MD, Williams CS, Payne CM, Dvorak B 2005 Epidermal growth factor reduces intestina apoptosis in an experimental model of necrotizing enterocolitis. Am J Physiol Gastrointest Liver Physiol 288:G755-G762

22. Leaphart CL, Cavallo J, Gribar SC, Cetin S, Li J, Branca MF, Dubowski TD, Sodhi CP, Hackam DJ 2007 A critical role for TLR4 in the pathogenesis of necrotizing enterocolitis by modulating intestinal injury and repair. J Immunol 179:4808-4820

23. Feng J, El-Assal ON, Besner GE 2006 Heparin-binding epidermal growth factor-like growth factor reduces intestinal apoptosis in neonatal rats with necrotizing enterocolitis. J Pediatr Surg 41:742-747; discussion 742-747

24. Caplan MS, Sun XM, Hseuh W, Hageman JR 1990 Role of platelet activating factor and tumor necrosis factor-alpha in neonatal necrotizing enterocolitis. J Pediatr $116: 960-964$
25. Giannone PJ, Nankervis CA, Richter JM, Schanbacher BL, Reber KM 2009 Prenatal lipopolysaccharide increases postnatal intestinal injury in a rat model of necrotizing enterocolitis. J Pediatr Gastroenterol Nutr 48:276-282

26. Caplan MS, Hedlund E, Adler L, Hsueh W 1994 Role of asphyxia and feeding in a neonatal rat model of necrotizing enterocolitis. Pediatr Pathol 14:1017-1028

27. Giannone PJ, Bauer JA, Schanbacher BL, Reber KM 2007 Effects of hyperoxia on postnatal intestinal development. Biotech Histochem 82:17-22

28. Giannone PJ, Schanbacher BL, Bauer JA, Reber KM 2006 Effects of prenatal lipopolysaccharide exposure on epithelial development and function in newborn rat intestine. J Pediatr Gastroenterol Nutr 43:284-290

29. Vicente AM, Guillen MI, Habib A, Alcaraz MJ 2003 Beneficial effects of heme oxygenase-1 up-regulation in the development of experimental inflammation induced by zymosan. J Pharmacol Exp Ther 307:1030-1037

30. Szabó C 2005 Pharmacological inhibition of poly(ADP-ribose) polymerase in cardiovascular disorders: future directions. Curr Vasc Pharmacol 3:301-303

31. Sánchez-Fidalgo S, Villegas I, Martín A, Sánchez-Hidalgo M, Alarcón de la Lastra C 2007 PARP inhibition reduces acute colonic inflammation in rats. Eur J Pharmacol 563:216-223

32. Beneke S 2008 Poly(ADP-ribose) polymerase activity in different pathologies-the link to inflammation and infarction. Exp Gerontol 43:605-614

33. Gerö D, Szabó C 2008 Poly(ADP-ribose) polymerase: a new therapeutic target? Curr Opin Anaesthesiol 21:111-121

34. Baregamian N, Rychahou PG, Hawkins HK, Evers BM, Chung DH 2007 Phosphatidylinositol 3-kinase pathway regulates hypoxia-inducible factor-1 to protect from intestinal injury during necrotizing enterocolitis. Surgery 142:295-302

35. Chokshi NK, Guner YS, Hunter CJ, Upperman JS, Grishin A, Ford HR 2008 The role of nitric oxide in intestinal epithelial injury and restitution in neonatal necrotizing enterocolitis. Semin Perinatol 32:92-99

36. Yu X, Radulescu A, Zorko N, Besner GE 2009 Heparin-binding EGF-like growth factor increases intestinal microvascular blood flow in necrotizing enterocolitis. Gastroenterology 137:221-230

37. Horwitz JR, Lally KP, Cheu HW, Vazquez WD, Grosfeld JL, Ziegler MM 1995 Complications after surgical intervention for necrotizing enterocolitis: a multicenter review. J Pediatr Surg 30:994-998; discussion 8-9

38. Hintz SR, Kendrick DE, Stoll BJ, Vohr BR, Fanaroff AA, Donovan EF, Poole WK, Blakely ML, Wright L, Higgins R; NICHD Neonatal Research Network 2005 Neurodevelopmental and growth outcomes of extremely low birth weight infants after necrotizing enterocolitis. Pediatrics 115:696-703

39. Rees CM, Pierro A, Eaton S 2007 Neurodevelopmental outcomes of neonates with medically and surgically treated necrotizing enterocolitis. Arch Dis Child Fetal Neonatal Ed 92:F193-F198 